\title{
Visual stimulus input, saccadic suppression, and detection of information from the postsaccade scene
}

\author{
EUGENE CHEKALUK and KEITH R. LLEWELLYN \\ University of New South Wales, Kensington, New South Wales, Australia
}

\begin{abstract}
Tachistoscopic presentation of saccadic stimulus sequences to fixating subjects produced saccadic suppression curves only when the pre- and postsaccade fixation fields were structured. Displacement in the sense either of movement of the intrasaccade display or of change from preto postsaccade fixation field was not required. Variation of intrasaccade displays from contours moving at saccadic rates to a stationary gray field had no effect. When one structured field immediately followed another, the change of sensitivity mimicked suppression, but an interposed grayout magnified the loss and therefore probably reduced carryover from the first fixation field. Interposed grayout also delayed recovery but reduced the latency of detection of information from the postfield, so that it appears to have reduced stimulus overload from the sudden presentation of the second field.
\end{abstract}

The saccadic movements that jerk the eye from one fixation point to another produce drastic changes in the visual input, which are, however, surprisingly free from obvious perceptual consequences. Paradoxically, the stimulus input itself is now recognized as being vitally important in protecting perceptual experience from its own potentially disruptive effects. These input effects are usually attributed to masking (see, e.g., Campbell \& Wurtz, 1978; Judge, Wurtz, \& Richmond, 1980; Matin, 1974), and attention has therefore generally been directed toward the pre- and postsaccade fixation fields as sources of forward and backward masking, respectively. In this paper, we shall concentrate on the third section of the stimulus sequence, the intervening intrasaccadic field. In particular, we shall deal with its effects on visual sensitivity and on detection of information in the postsaccade period.

Thinking about the intrasaccadic field has been dominated by the fact that it is characterized by the very fast motion of image features over the retina. At one extreme, this rapid image motion (or, alternatively, the rapid changes of luminance that such motion would produce at retinal loci) has been nominated as the sole source of saccadic suppression (Mateeff, Yakimoff, \& Mitrani, 1976). At the other extreme, the intrasaccade period has tended to be denied a role, because the image motion is consi-

All experiments were carried out by E. Chekaluk-Experiment 1 in partial fulfillment of a BA with honors at the University of New South Wales, and the remaining experiments in partial fulfilment of. K. R. Llewellyn acted as supervisor throughout. Requests for reprints should be sent to Eugene Chekaluk, who is now at the Department of Behavioural Sciences, Cumberland College of Health Sciences, University of Sydney, P.O. Box 170, Lidcombe, NSW, 2141, Australia. dered to be so fast that contour motion would become ineffective. MacKay (1973), for example, attributes suppression to the neural shock produced by the sudden replacement of the structured presaccade field by the structured postsaccade field. The appearance of the intrasaccade input when it is made visible (e.g., by limiting illumination to this period) supports the view that the moving contours would be ineffective, because structured displays such as a page of print (Dodge, 1900) or a Snellen Chart (Campbell \& Wurtz, 1978) become unreadable fused blurs-an effect that Campbell and Wurtz referred to as grayout.

During their investigations of the nondetection of grayout, Campbell and Wurtz (1978) and Corfield, Frosdick, and Campbell (1978) made use of stimulus conditions in which rapidly moving contours were eliminated. Fixating subjects were presented with stimulus sequences in which stationary blank displays of saccadic duration represented the grayout. Campbell and Wurtz found that the masking of structured pre- and postsaccade fixation fields by this grayout substitute decreased as a function of the duration of the structured field. They argued that this was consistent with the effect of postsaccade field duration on the masking of saccadic grayout.

Campbell and Wurtz (1978) were concerned with just one of the problems associated with saccades-the nonexperience of the grayout (which they called saccadic omission). This question can be explored most directly by using the actual grayout as a detection target, and for this a perceptual equivalence between saccadic grayout and a blank field as targets may be sufficient. This type of study, however, differs from the usual saccadic suppression experiment, in which visual sensitivity is tracked throughout the whole saccade sequence, including the extrasaccade periods. For such studies, one would have 
to show that the stationary blank field is functionally, as well as perceptually, equivalent to normal grayout input.

The difference between the perceptual effect of the rapid image motion and its possible functional role has been pointed out by Matin $(1974,1976)$, who noted that some Y ce Is respond to stimulus velocities well above thresholds for seen movement. Such cells could allow the rapidly moving contours to affect sensitivity despite the fused, grayout perceptual nature of this input. Matin suggested that the combination of stimulation from the rapidly moving contours of the intrasaccadic period and masking from the static fixation fields may be particularly powerful. A similar view has been expressed by Breitmeyer and Ganz (1976).

In Experiments 1 and 2, we tested whether intrasaccadic input contributes to changes in sensitivity through its dynamic features either directly or by means of interaction with the extrasaccade input. This leaves a further question, however. Even if the dynamic property of the intrasaccadic period is ineffective, it has another property: It is always present in saccades. Cumming (1978) has suggested that its presence is important in maintaining perceptual stability. As regards sensitivity changes, the intrasaccade period represents a brief separation between the fixation fields, and it could, perhaps, serve as a buffer in reducing the neural shock postulated by MacKay (1973). It could also serve to limit interference by the original fixation scene to the perception of the new scene by providing time during which backward masking of the old scene by the new may operate. Since this period is always present in normal saccades, research into this question requires the use of a modified version of the technique developed by MacKay (1970), in which the saccadic stimulus sequence is presented to a fixating subject. In Experiments 1 and 2, a sequence corresponding to pre-, intra-, and postsaccade input was tachistoscopically presented to a fixating subject. In Experiments 3, 4, and 5 , this procedure was modified so that the standard sequence was compared to one in which the postsaccade display immediately followed the presaccade display with no intervening intrasaccade display.

In Experiments 1-4, the traditional saccadic suppression procedure with a probe stimulus was used, to investigate changes in visual sensitivity. The usual assumption appears to be that our response to the new fixation field will be more efficient if recovery from suppression is rapid. In Experiment 5, we directly tested the efficiency of information extraction in this period, using a latency of discrimination technique.

\section{EXPERIMENT 1}

The effect of the dynamic aspects of the intrasaccade input on sensitivity may be directly tested by comparing a stationary gray field with contours moving at saccadic rates. Masking by extrasaccade stimuli may be examined by comparing a highly structured pre-/postsaccade field with a pre-/postsaccade field in which the only structure is a fixation point on a white background. The use of a factorial design allows evaluation of the suggestion by Matin $(1974,1976)$ that the combination of extrasaccade and intrasaccade features provides the optimal masking situation

\section{Method}

Stimuli. Stimuli were drawn on standard tachistoscope cards subtending $6^{\circ}$ vertical $\times 9^{\circ}$ horizontal. The same pattern was used for both the pre- and the postsaccade field (either plain for both or pattern for both). The pattern stimulus was a grid of black and white vertical and horizontal square-wave gratings. Both the verti$\mathrm{cal}$ and the horizontal gratings had frequencies of $0.5 \mathrm{cpd}$ of visual angle. Both the pre- and the postsaccade displays had a central $0.25^{\circ}$ black fixation point.

The intrasaccade displays were either blank (a grayout substitute consisting of a stationary card of a uniform midgray) or moving (a contoured display of black and white stripes moving from right to left at approximately $400 \% \mathrm{sec}$ ). The stripes formed a continuous vertical square-wave grating with a frequency of $0.5 \mathrm{cpd}$.

The target was a circular patch of horizontal square-wave grating, $2^{\circ}$ in diameter, centered on a white card (and thus centered on the pre-/postsaccade fixation point). The black and white stripes had a frequency of $2.5 \mathrm{cpd}$ of visual angle.

Under the channel illumination setting used for the pre- and postsaccade fields in this and all subsequent experiments, the luminance of the white areas of the displays was approximately $8.5 \mathrm{~cd} / \mathrm{m}^{2}$, that of black areas $0.3 \mathrm{~cd} / \mathrm{m}^{2}$, and that of the gray card $5.8 \mathrm{~cd} / \mathrm{m}^{2}$. Flicker photometry showed that the gray card approximated the brightness of the rapidly moving stripes. During rest periods, the subject was exposed to the lighting of the experimental room, and the luminance varied from around $7 \mathrm{~cd} / \mathrm{m}^{2}$ for the outside surface of the tachistoscope down to about $0.7 \mathrm{~cd} / \mathrm{m}^{2}$ for the black walls.

Stimulus sequence. The 1.5 -sec presaccade field was followed by the $50-\mathrm{msec}$ intrasaccade field and then by the 1.5 -sec postsaccade field. When present, the target replaced the current display for $10 \mathrm{msec}$. Onset time (relative to intrasaccade field onset) was varied as follows: 80 or $40 \mathrm{msec}$ before onset of the intrasaccade field, or 20,90 , or $130 \mathrm{msec}$ after its onset. For the 20 -msec-afteronset condition, the target occupied the middle $10 \mathrm{msec}$ of the 50 -msec intrasaccade period.

Stimulus presentation. Stimuli were presented by means of a three-channel tachistoscope (Gerbrands Model T-3B-1) controlled by a 6809 microcomputer. The intrasaccade field contours were black and white stripes on an endless belt mounted directly behind a $6^{\circ} \times 9^{\circ}$ window at the end of one channel. The $0.1865 \mathrm{~kW}$ electric motor driving the belt was kept running throughout each experimental session. The blank stimulus was mounted on a metal plate that could be slid on a set of guides between the moving belt and the window. When that channel was illuminated, therefore, either the rapidly moving contours or the interposed stationary gray field was presented

Subjects and Procedure. Nine unpaid volunteers from an introductory psychology course were individually tested. They were instructed to fixate the center of the presaccade field and to indicate, by means of a 4-point scale (yes, certain; yes, uncertain; no, uncertain; no, certain), their confidence that the target had or had not been presented. Each subject received approximately 24 practice trials with the plain/blank/plain sequence, target onset being $20 \mathrm{msec}$ after intrasaccade field onset. The target intensity was adjusted for each subject to give approximately $75 \%$ "yes, certain" responses when the target was present.

The subjects were then given 1,000 test trials, arranged in two blocks of 500 . The pre-/postsaccade field was varied over blocks, and the intrasaccade field and target onset times were varied within blocks. Each of the 10 intrasaccade field/target onset time combinations was presented on 25 trials within a block, the remaining 250 trials being no-target trials. Order of blocks and order of con- 
ditions within blocks were randomized. The intertrial interval was controlled by the subject.

\section{Results}

Ratings for each onset time were converted into $d^{\prime}{ }_{e}$ values. This measure is $d^{\prime}$ (the difference between the means of the signal and noise distributions) expressed in units equal to the average of the standard deviations of the signal and noise distributions (rather than of one standard deviation or the other). The four-category rating scale was that recommended by McNicol $(1972,1984)$, and the Torgerson method of calculation used may be found in McNicol (1984). The mean $d^{\prime}{ }_{e}$ values are graphed as Figure 1.

The data were analyzed by means of three planned contrasts, for which the Bonferroni procedure was used to adjust the alpha value for the family of contrasts to .05 . The variation of the pre-/postsaccade field from unstructured (plain) to highly structured (pattern) significantly lowered sensitivity $[F(.05 / 3 ; 1,8)=18.602, p=.002]$. The variation of the intrasaccade field from rapidly moving contours to stationary gray field had no significant effect on sensitivity $[F(.05 / 3 ; 1,8)=0.086, p=.777$, which exceeds $.05 / \mathbf{k}$, where $\mathbf{k}=$ number of contrasts $=$ 3]. There was no significant interaction effect between the pre-/postsaccade and intrasaccade field conditions $[F(.05 / 3 ; 1,8)=3.168, p=.113]$.

\section{Discussion}

The statistical results are supported by the detailed time courses shown in Figure 1. When structured pre-/post-

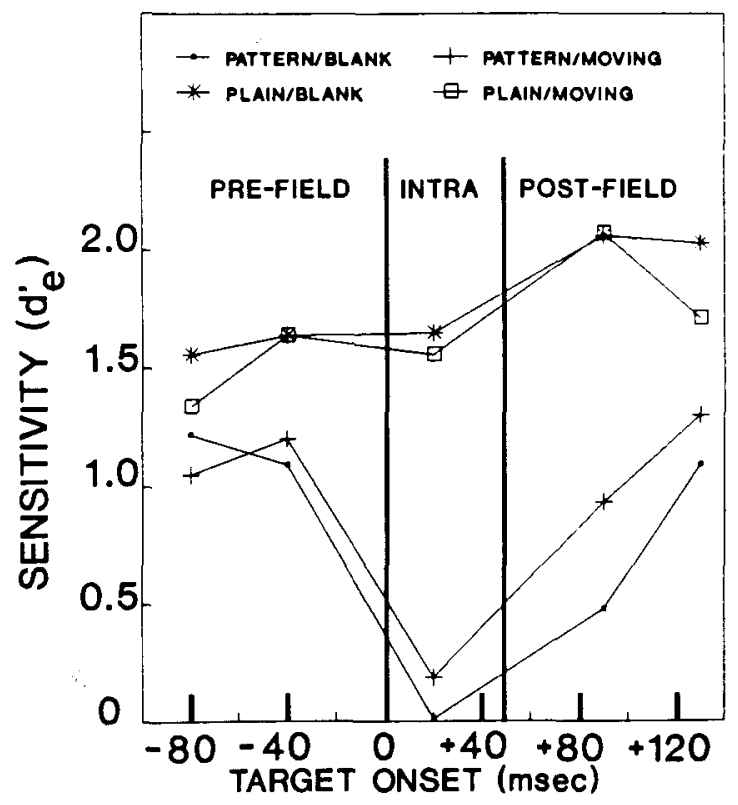

Figure 1. Sensitivity means for the combinations of structured and nonstructured pre- and postsaccade fixation fields, with intrasaccade fixation fields consisting of either contours moving at a saccadic rate or a stationary gray field. saccade fields are present, a curve of the saccadic suppression type is obtained, regardless of the presence or absence of rapidly moving contours in the intrasaccade period. When the pre-/postsaccade fields are almost totally devoid of structure, no such curves are obtained (again regardless of intrasaccade field condition). The effect of the structured pre-/postsaccade fields is not enhanced in any way by the presence of rapid contour motion in the intrasaccade period.

The blank condition did not involve any rapid displacement in the sense of motion of a structured retinal image, and, since the presaccade and postsaccade fields were identical, there was no sudden displacement of the position of details. Explanations of the effect of stimulus input upon sensitivity that are based on displacement in either sense (e.g., MacKay, 1970, 1973) therefore appear not to hold, for displacement is not a necessary component of the stimulus sequence.

The rapid contour display did, however, lack the normal jerk of stationary contours into motion, and this could perhaps account for its equivalence to the blank (see, e.g., Breitmeyer, 1980; MacKay, 1973). To test this possibility, 6 subjects were given 200 trials in which the striped belt from Experiment 1 was presented as stationary for $1.5 \mathrm{sec}$ and the motor was then turned on for $50 \mathrm{msec}$. The display was then viewed until a further $1.5 \mathrm{sec}$ had elapsed. The target from Experiment 1 was presented on half the trials for $10 \mathrm{msec}$ in the middle of the motor-on period. These jerk conditions were then compared to a blank condition, in which the gray card was interposed between the stationary stripe displays for $50 \mathrm{msec}$. No significant differences in sensitivity were found. Although no measures of the nature and duration of the jerked motion produced by the 50 -msec activation of the (abused) motor were taken, the lack of significant effects suggests that the absence of such a jerk does not account for the results of Experiment 1.

Our negative results could reflect our use of naive subjects, which may have led to an increase in error variance with a subsequent loss of statistical power. The number of trials $(1,000)$ and the number of subjects $(9)$ should have been sufficient to overcome this, but we increased the number of subjects tested on the appropriate conditions (movement versus blank with unstructured extrasaccade fields) to 17. No significant effect was found.

A further possible objection is that our results for the structured extrasaccade conditions do not show the characteristic drop in sensitivity before the onset of the intrasaccade period. This failure was attributed to the particular onset times used, and this interpretation was tested in Experiment 2.

\section{EXPERIMENT 2}

\section{Method}

Stimuli and presentation. The stimulus sequence pattern/blank/pattern was used in conjunction with the patterned target. The blank and the target were produced by the tachistoscope cards used in Experiment 1, while the structured pre-/postsaccade 


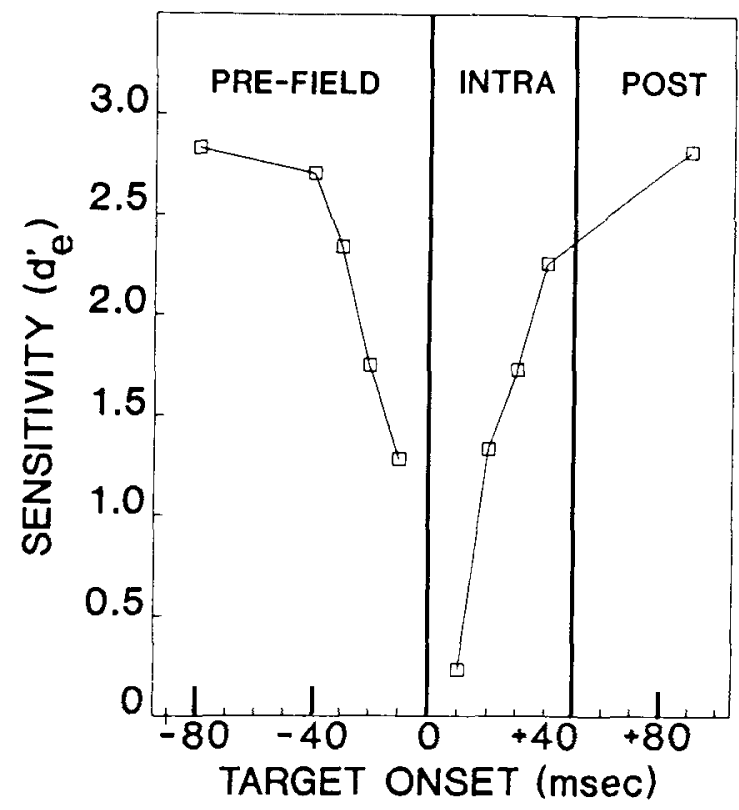

Figure 2. Time course of sensitivity for a sequence of structured presaccade fixation field, stationary gray intrafield, structured postsaccade fixation field, showing the drop in sensitivity preceding intrasaccade fixation field onset.

display was a similar card containing a vertical square-wave grating of black and white stripes, having a spatial frequency of $0.5 \mathrm{cpd}$. Stimulus durations were as those in Experiment 1. A more detailed time course was obtained by using target onset times of 80,40 , 30,20 , and $10 \mathrm{msec}$ before the onset of the blank, and $10,20,30$, 40 , and $90 \mathrm{msec}$ after its onset.

Subjects and Procedure. Four postgraduate students from the School of Psychology were instructed in the use of the response scale and then given 20-25 practice trials, followed by 500 randomized test trials. On 250 of the test trials, the target was not presented, and on the other 250 trials, the target was presented for 25 trials for each of the 10 target times. The intertrial interval was controlled by the subject.

\section{Results}

Mean $d^{\prime}{ }_{e}$ values for the group of subjects are presented in Figure 2. The drop in visual sensitivity preceding the onset of the blank was tested by comparing the mean $d^{\prime}{ }_{e}$ values for 80 and $10 \mathrm{msec}$ before blank onset. The drop was significant $[F(1,3)=39.6, p=.008]$.

\section{Discussion}

Allowing for the effects on the saccadic suppression curve related to target intensity (and of limitations imposed by the specific times used), the results presented in Figure 2 conform closely to the general pattern of results from other studies (e.g., Latour, 1962; Volkmann, Schick, \& Riggs, 1968). The presaccade drop in sensitivity normally associated with saccadic suppression curves is clearly seen. Recovery is well under way by the end of the intrasaccade period.

Experiments 1 and 2 show that the visual sensitivity change produced by contours in the environment arises from the fixation periods. The role of structure in these periods is consistent with the view that the process is masking.

As noted earlier, it is possible that the normally unavoidable intrasaccade period could still play an important role. It provides onsets and offsets that cause inhibition of sustained channel activity by transient channels. It could act as a delay period in which various masking factors have time to operate. Its presence may actually overcome the disruptive effects suggested by MacKay (1973), which might otherwise arise from very rapid displacement. Although the intrasaccade period can never be eliminated from a saccadic eye movement, the technique of presenting its stimulus equivalents to a fixating eye enables us to examine the consequences of its presence and absence.

\section{EXPERIMENT 3}

A limited time course was obtained using the structuredpresaccade-field/blank/structured-postsaccade-field sequence. This was compared with the time course when the blank intrasaccade field was omitted and the second structured field followed immediately upon the first.

\section{Method}

Stimuli. The structured pre- and postsaccade fields were squarewave gratings of $0.5 \mathrm{cpd}$. The grating was either vertical or horizontal, and the postsaccade field grating was always orthogonal to that used as the presaccade field. The target was centered on a white background and was a $2^{\circ}$ diameter circular field of square-wave grating. Its frequency was $2.5 \mathrm{cpd}$, and its orientation was randomly selected from either $45^{\circ}$ left or $45^{\circ}$ right.

Stimulus sequence. Presaccade and postsaccade fixation field durations were both $200 \mathrm{msec}$, and, when present, the intrasaccade duration was $50 \mathrm{msec}$. Target duration was $10 \mathrm{msec}$, and it was presented $50 \mathrm{msec}$ before the offset of the presaccade fixation field, $40 \mathrm{msec}$ after the onset of the postsaccade fixation field, or in the middle of the stimulus sequence (i.e., $20 \mathrm{msec}$ after the onset of the gray intrasaccade field in the grayout-present condition or immediately after presaccade field offset in the grayout-absent condition)

Subjects and Procedure. Forty volunteers from an introductory course in psychology acted as subjects. The procedure was as described previously, and a total of 600 test trials (half with target absent) was used. The presentation of the vertical/horizontal and horizontal/vertical extrasaccade field pairings was counterbalanced over subjects.

\section{Results}

Mean $d^{\prime}{ }_{e}$ values are shown in Figure 3. A planned contrast over groups showed that the order of presentation of vertically and horizontally striped extrasaccade fields had no effect $[F(1,38)=0.797, p=.378]$. Planned contrasts carried out over repeats showed that the average sensitivity over the three target onset conditions did not differ for the grayout-present and grayout-absent conditions $[F(.05 / 2 ; 1,38)=3.556, p=.067, p>.05 / 2]$. The mean sensitivity for the middle target onset condition averaged over both grayout conditions was significantly less than the average sensitivity over the other two onset conditions $[F(.05 / 2 ; 1,38)=65.12, p$ approximates zero]. 


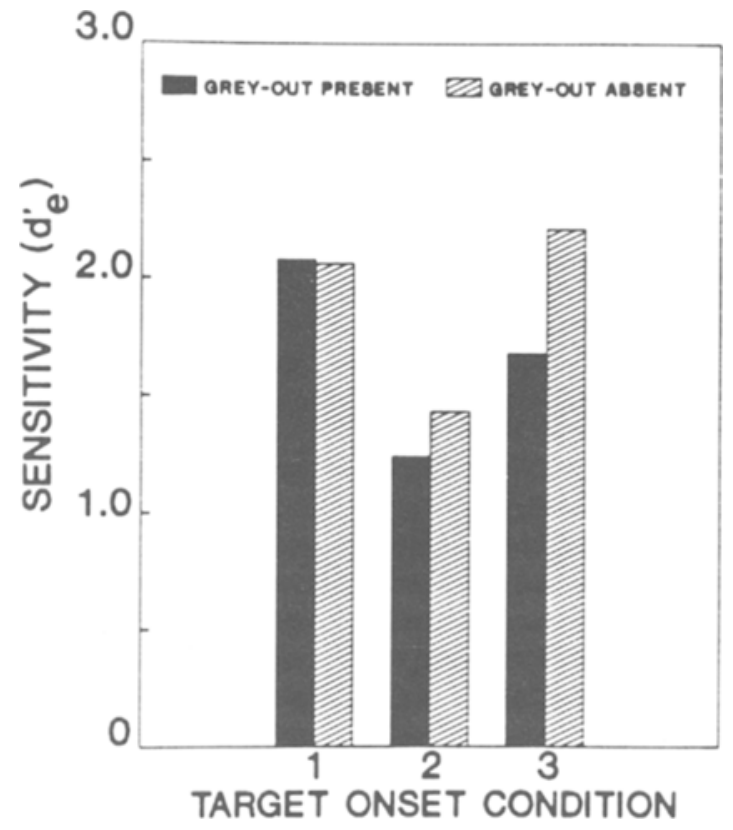

Figure 3. Sensitivity means when the gray field is present or absent from the stimulus sequence. Target onsets were (1) $50 \mathrm{msec}$ before the offset of the presaccade fixation field; (2) 20 msec after grayout onset, or, when grayout absent, immediately after presaccade fixation field offset; and (3) $\mathbf{4 0} \mathbf{~ m s e c}$ after postsaccade fixation field onset.

The differences between the grayout-present and grayoutabsent conditions apparent in the recovery phase of the sensitivity curve (target onset $20 \mathrm{msec}$ after postsaccade field onset) were tested by a post hoc contrast. The presence of the grayout led to less recovery $[F(1,38)=$ 9.149, $p=.004]$.

\section{Discussion}

The results shown in Figure 3 suggest that the grayout period is not an essential aspect of the changes in sensitivity associated with saccades, because the usual pattern of sensitivity change is obtained in its absence. It also does not function in a way that enhances rapid recovery of sensitivity in preparation for the postsaccade fixation field; indeed, its presence appears to slow down this recovery. This slowdown is all the more remarkable in that the target was actually presented $50 \mathrm{msec}$ later in the grayoutpresent condition than in the corresponding grayout-absent condition, relative to the offset of the presaccade fixation field. In other words, the onset of the target at $40 \mathrm{msec}$ following the onset of the postsaccade fixation field was also $40 \mathrm{msec}$ after the offset of the presaccade-field in the grayout-absent condition, but it was $90 \mathrm{msec}$ after this offset in the grayout-present condition, because the $50 \mathrm{msec}$ of grayout intervened.

\section{EXPERIMENT 4}

The different time courses of sensitivity change for the grayout-present and -absent conditions were examined in more detail in the following experiment. One of the authors (E.C.) served as the subject.

\section{Method}

The only change from Experiment 3 was that the number of target onset times was extended. The middle target onset time was retained (i.e., $20 \mathrm{msec}$ after grayout onset in the grayout-present condition and immediately after presaccade field offset for the grayout-absent condition). The other onset times were 50 and $10 \mathrm{msec}$ before presaccade field offset and 10, 40,70, and $100 \mathrm{msec}$ after postsaccade field onset.

\section{Results and Discussion}

The results are presented in Figure 4. These data are consistent with those of the previous experiment in that recovery is actually slower when the grayout is present in the stimulus sequence. The degree of reduction of sensitivity was subsequently measured by an adjustment procedure (the subject set the target intensity over a number of repetitions of each condition), and the maximum elevation of threshold when grayout was present was found to be approximately $1.2 \mathrm{log}$ units (for the target in the middle of the grayout period). Maximum threshold elevation for the grayout-absent condition was $0.8 \mathrm{log}$ units.

The presence of grayout both maximizes the loss of sensitivity and slows recovery. As regards the observer's sensitivity to features of the postsaccadic fixation scene, it could, therefore, be seen as a negative factor, but in functional terms the importance of the new scene lies in the new information it provides. Although we obviously cannot process information if it cannot be detected by the visual system, information detection may be more efficient if the stimulus input is more gradual. Although the sudden onset of the postsaccade field (as in the grayoutabsent case) is not disruptive to the recovery of sensitiv-

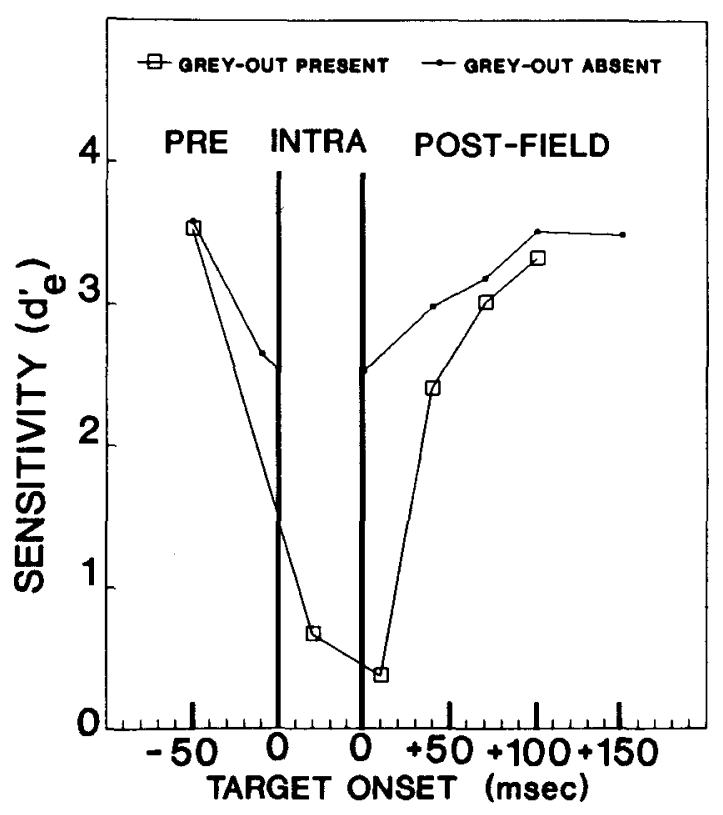

Figure 4. Sensitivity timecourse for Subject E.C., with grayout present or absent. 


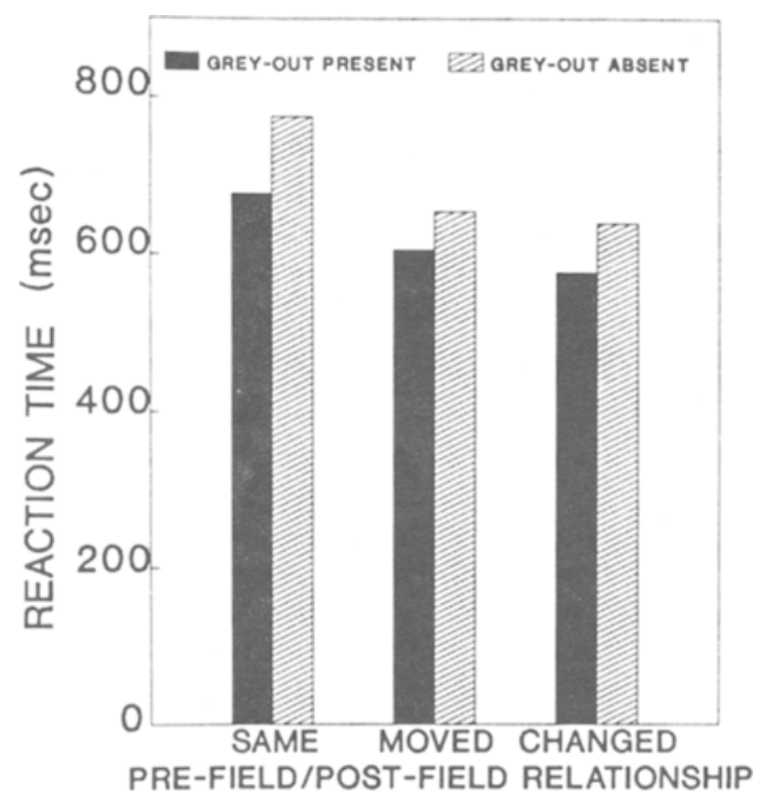

Figure 5. Mean reaction times for the detection of whether the postsaccade fixation field was the same as the presaccade fixation field, moved (a displaced version), or changed (a different display).

ity, it may be disruptive to the processing of the information contained in the new sensory input. Experiment 5 was designed to examine this possibility.

\section{EXPERIMENT 5}

The possibility that the grayout may have a positive functional role (limiting stimulus overload from the postsaccadic fixation scene) was examined in Experiment 5 by changing the subject's task from that of detecting a target to that of extracting some basic information from the postgrayout scene.

\section{Method}

Stimuli. The prefield was a white card on which a vertical squarewave grating of $0.5 \mathrm{cpd}$ was present in either the left or the right half. The postsaccade-fixation field was the same display, a similar display in which the pattern was displaced (from the left to the right or vice versa), or a different display (a card totally covered with similar black and white stripes oriented horizontally). The presaccade fixation field was presented for $1.5 \mathrm{sec}$, but the postsaccade duration was limited to $10 \mathrm{msec}$. The $50-\mathrm{msec}$ grayout field was the gray card used previously.

Subjects and Procedure. Ten volunteers from an introductory course in psychology were given 24 practice trials and 300 test trials. On half the trials, the pre- and postsaccade fields were separated by the presentation of the gray intrasaccade field. The subjects were required to indicate which postsaccade field was presented, as quickly as possible, by pressing one of three buttons. All conditions were randomized.

\section{Results}

Mean reaction times for the six conditions are shown in Figure 5. A planned contrast showed that, overall, the presence of grayout led to a significantly faster identifi- cation reaction time than did its absence $[F(.05 ; 1,9)=$ $7.11, p=.026$ ]. Planned contrasts (adjusted to an alpha level of .05) showed significant differences between the three types of postsaccade fixation field. The identical postsaccade field took significantly longer to identify than did the average of the other two postsaccade fields $[F(.05 / 2 ; 1,9)=8.463, p=.017]$, whereas the other two did not differ from each other $[F(.05 / 2 ; 1,9)=1.461, p=$ $.258, p>.05 / 2]$.

\section{Discussion}

The one significant effect due to the nature of the postsaccade fixation fields shows that subjects find it easier to detect that a change has occurred rather than not. The major finding is that the identification of the postsaccade scene is more rapid if the grayout is included in the stimulus sequence. Since its presence reduces sensitivity in the postsaccade period, it is reasonable to suppose that a positive effect on information detection results from the limitation of the sensory overload that would normally be expected to occur with the sudden onset of the second fixation scene. The degree of sensitivity loss is increased when the grayout is present, so that it may be making a further contribution through the minimization of carryover from the presaccade fixation scene. In other words, it may be helping "to wipe the slate clean."

\section{GENERAL DISCUSSION}

The most dramatic feature of the stimulus sequence associated with saccades is the rapid image motion during the intrasaccade period. Indeed, the term (rapid) image motion has been used to distinguish stimulus contributions from central mechanisms (see, e.g., Howard, 1982; Wolf, Hauske, \& Lupp, 1978). We have, however, failed to detect any effect that rapidly moving contours might have on saccadic suppression. The presence of such dynamic stimulus features is not a necessary property of the stimulus sequence (Experiments 1 and 2), nor does it contribute to the changes in sensitivity, either directly or through interaction (Experiment 1). Displacement in this sense of motion or, alternatively, of change of position is not a necessary feature of the stimulus sequence. The essential aspect is the presence of structured stimulation corresponding to input during fixations.

A possible objection to our results is that, although subjects were always instructed to fixate and were given control of trial onset, we did not monitor eye movements. The effects of any spontaneous saccades that might have occurred would have to be considered. Any effects due to central inhibition would presumably be equal over all extrasaccade stimulus conditions, and since the saccades could occur at any time during the presentation of a stimulus sequence, there would simply be an overall reduction in measured sensitivity levels. Unless the number of such spontaneous saccades was high, these effects would be expected to be minimal, because we used large numbers of trials and, in all but Experiment 4, group data. Any stimulus effects produced by spontaneous saccades dur- 
ing the extrasaccade periods would depend on the presence or absence of structure. Although other interactions with target visibility are possible, the most likely effect would be a weakening of the contribution of the structured field because of its partial masking. The effect of such saccades would therefore be to obscure differences between conditions. Our results suggest that this did not pose a problem.

Although we found no evidence to support Matin's suggestion of a direct effect of rapidly moving intrasaccadic stimulation (Matin, 1974, 1976), the combination of such rapid motion with extrasaccade structure could, as she suggests, be particularly potent. Rapid image motion reduces structured intrasaccade fields to degraded blurs, which may represent optimal targets for masking. Campbell and Wurtz (1978) have stressed the need for such smearing if effective masking is to take place. The traditional emphasis on metacontrast masking (involving the interaction of contours) has tended to direct attention away from the possible contribution of the smearing of the intrasaccade image to its own masking, and, in fact, led Corfield et al. (1978) in their companion paper to suggest that masking was not involved in saccadic omission, because classical masking does not apply to blanks. Masking of contourless areas has, however, been demonstrated by Stoper and Mansfield (1978). In our experiments, we did not take systematic records of the visibility of the grayout display, but the general effect of our stimulus sequence presentations appeared to be the equivalent of saccadic omission.

Evidence suggests that the mechanism postulated by the rival central inhibition theory, an inhibition linked to the eye movement control center, does exist (see, e.g., Richmond \& Wurtz, 1980; Riggs, Merton, \& Morton, 1974; Riggs, Volkmann, Moore, \& Ellicot, 1982), although Brooks and Fuchs (1975) and Brooks, Impelman, and Lum (1981) have failed to obtain threshold elevation at low background luminance levels. Its role, however, is now more likely to be considered to be that of supplementing stimulus effects. A common position is that first put forward by Bridgeman (1977), who suggested that central inhibition takes over when stimulus factors are weak (as in scotopic conditions). The results of Experiment 1 may appear to support this view, since no evidence of suppression was found when spatial frequency was zero. Threshold elevation apparently due to noncentral effects has, however, been found with blank fields by Brooks and Fuchs (1975) and Brooks et al. (1981). The discrepancy between our results and those of Brooks et al. (1981) is probably due to the fact that our tachistoscopic presentation did not involve the change in the angle of incident light rays to the receptors to which they attribute the effect.

The view that stimulus effects have to be replaced or supplemented by central mechanisms is often very broadly expressed. In this paper, we have concentrated on the specific issues of sensitivity changes and of extraction of information from the new scene. In this context, neither the views of Bridgeman (1977) nor the findings of Brooks et al. (1981) appear to be particularly relevant. Bridgeman was concerned with the maintenance of subjective stability when stimulus factors are weak, while the results of Brooks et al. relate to the question of whether it is actually necessary to postulate central inhibition. With respect to the specific issues of sensitivity loss and detection of information in new scenes, it would seem that featureless or completely dark fields pose no problem for the perceptual system (what input has to be suppressed?) and that dimly seen features are probably only a minimal problem. There is also little information to be detected in the new fixation field.

Volkmann, Riggs, White, and Moore (1978) suggested that supplementation by central inhibition may be required throughout the full range of illumination. Using severely restricted stimulus conditions, they found a marked loss of contrast sensitivity associated with low spatial frequencies. They argued that this apparently central effect would compensate for a loss of masking from stimulus input with decreasing contour information in the visual field (decreasing spatial frequency). Our structured fields were, however, of low spatial frequency (between the two lowest frequencies used by Volkmann et al.) and they appeared to be quite effective. Similarly, Corfield et al. (1978) found that the effectiveness of pre- and postsaccade fields in eliminating grayout actually increased as their spatial frequency decreased. The argument that stimulus effects require supplementation at low spatial frequencies, therefore, does not seem to hold. The similarity in the size of threshold elevation obtained when the saccadic movement is absent (i.e., a field is jerked at saccadic rates) to those obtained when a saccade is made (see, e.g., Brooks \& Fuchs, 1975) also brings into question the existence of supplementation. The question of the role of central inhibition has therefore still to be resolved.

In general, our results are in agreement with those of MacKay (1970), Brooks and Fuchs (1975), Brooks, Impelman, and Lum (1981) and others who have emphasized the importance of stimulus input. They also support the assertion by Woodworth (1906) that there should be no difference in perception between the saccading and the stationary eye, provided that the stimulus conditions are equivalent. They do, however, raise the question of what is necessary for such equivalence. The standard procedure is to present a fixating subject with a field that is jerked at saccadic rates. Our results show that this movement is not necessary, for the temporal sequence of structured field/blank/structured field is sufficient to reproduce saccadic suppression curves. This is the case even when the blank merely serves as a brief interruption to the otherwise continuous presentation of the same structured field. If the structured fields differ, the sequence structured field/structured field is sufficient to produce an effect. The intrasaccadic input is not a necessary part of the sequence, but it serves to magnify the effect. The properties of the grayout that may be responsible for this 
magnification are the provision of an extrasaccade field change within a very brief timespan and the provision of time for masking processes to operate.

In summary, our results show that we are in the fortunate situation in which the normally unavoidable presence of the grayout helps to satisfy the purpose of the original saccade by speeding the extraction of information from the new scene.

\section{REFERENCES}

Breitmeyer, B. G. (1980). Unmasking visual masking: A look at the "why" behind the veil of the "how." Psychological Review, 87, $52-69$.

BreitMEYeR, B. G., \& GANZ, L. (1976). Implications of sustained and transient channels for theories of visual pattern masking, saccadic suppression and information processing. Psychological Review, 83, 1-36.

Bridgeman, B. (1977). Reply to Brooks and Fuchs: Exogenous and endogenous contributions to saccadic suppression. Vision Research, 17, 323-324

Brooks, B. A., Impelman, D. M. K., \& LUM, J. T. (1981). Backward and forward masking associated with saccadic eye movement. Perception \& Psychophysics, 30, 62-70.

Brooks, B. A., \& Fuchs, A. (1975). The influence of stimulus parameters on visual sensitivity during saccadic eye movements. $\mathrm{Vi}$ sion Research, 15, 1389-1398.

Campbell, F. W., \& Wurtz, R. H. (1978). Saccadic omission: Why we do not see a grey-out during a saccadic eye movement. Vision Research, 18, 1297-1303.

Corfield, R., Frosdick, J. P., \& Campbe1., F. W. (1978). Grey-out elimination: The roles of spatial waveform, frequency and phase. $V_{i-}$ sion Research, 18, 1305-1311.

Cumming, G. D. (1978). Eye movements and visual perception. In E. C. Carterette \& M. P. Friedman (Eds.), Handbook of perception: Vol. 9. Perceptual processing (pp. 221-255). New York: Academic Press.

Dodge, R. (1900). Visual perception during eye movement. Psychological Review, 7, 454-465.

HoWARD, I. P. (1982). Human visual orientation. New York: Wiley.

JUdGE, E. J., WURTZ, R. H., \& RICHMOND, B. J. (1980). Vision during saccadic eye movements: $I$. Visual interactions in striate cortex. Journal of Neurophysiology, 43, 1135-1155.

Latour, P. L. (1962). Visual threshold during eye movements. Vision Research, 2, 261-262.
MACKAY, D. M. (1970). Elevation of visual threshold by displacement of retinal image. Nature, 2, 90-92.

MACKAY, D. M. (1973). Visual stability and voluntary eye movements. In R. Jung (Ed.), Handbook of sensory physiology (Vol. 7, Pt. 3, pp. 307-331). Berlin: Springer-Verlag.

MATEefF, S., YAkimofF, N., \& Mitrani, L. (1976). Some characteristics of the visual masking by moving contours. Vision Research, 16, 489-492.

Matin, E. (1974). Saccadic suppression: A review and an analysis. Psychological Bulletin, 81, 889-917.

Matin, E. (1976). Saccadic suppression and the stable world. In R. A. Monty \& J. W. Senders (Eds.), Eye movements and psychological processes (pp. 113-119). Hillsdale, NJ: Erlbaum

MCNicOL, D. (1972). A primer of signal detection theory. London: Allen \& Unwin.

MCNicol, D. (1984). The use of signal detection theory in the evaluation of information displays. In R. Easterby \& H. Zwaba (Eds.) Information design (pp. 91-126). Chichester, England: Wiley.

Richmond, B. J., \& WuRTz, R. H. (1980). Vision during saccadic eye movements: II. A corollary discharge to monkey superior colliculus. Journal of Neurophysiology, 43, 1156-1167.

Riggs, L. A., Merton, P., \& Morton, H. (1974). Suppression of visual phosphenes during saccadic eye movements. Vision Research, 14, 997-1011.

Riggs, L. A., Volkmann, F. C., Moore, R. K., \& Ellicotr, A. G. (1982). Perception of suprathreshold stimuli during saccadic eye movement. Vision Research, 22, 423-428.

Stoper, A. E., \& MANSFIEld, J. G. (1978). Metacontrast and paracontrast suppression of a contourless area. Vision Research, 18, 1669-1674.

Volkmann, F. C., Riggs, L. A., White, K. D., \& Moore, R. K. (1978). Contrast sensitivity during saccadic eye movements. Vision Research, 18, 1139-1199.

Volkmann, F. C., Schick, A. M. L., \& Riggs, L. A. (1968). Time course of visual inhibition during voluntary saccades. Journal of the Optical Society of America, 58, 562-569.

WOLF, W., HaUSKe, G., \& LuPP, U. (1978). How presaccadic gratings modify postsaccadic modulation transfer function. Vision Research, 18, 1173-1179.

WOODWORTH, R. (1906). Vision and localization during eye movements. Psychological Bulletin, 3, 68-70.

(Manuscript received January 29, 1988; revision accepted for publication February 14, 1990.) 